\title{
Episodic time in the brain: A new world order
}

\author{
Catalin V. Buhusi ${ }^{1}$ \\ Published online: 3 April 2019 \\ (C) The Psychonomic Society, Inc. 2019
}

\section{Summary}

Recent findings from the laboratory of May-Britt and Edvard Moser (Tsao et al. in Nature 561, 57-62, 2018) suggest that episodic time is integrated from experience by a neural population in lateral entorhinal cortex that encodes events in a whenwhere-what trajectory at multiple time scales. While they provide a window into how the brain represents episodic memory, these findings also raise questions about whether this when-where-what trajectory truly reflects the temporal order of events, or whether one needs additional pieces of information to reconstruct temporal order, possibly using either associative information or evolutionary asymmetries built into biochemistry or neural circuits.

Keywords Episodic memory $\cdot$ Timing $\cdot$ Navigation $\cdot$ Spatial learning $\cdot$ Brain $\cdot$ Entorhinal cortex

How do we make sense of the world? How do we represent the world in our brains? How do we place ourselves and objects around us in our constructed representations of the world? With regard to visual stimuli, one creates in the brain a map resembling the world. Similarly, we seamlessly encode snippets of when-where-what information in episodic memory, reflecting personal experiences that occurred at a particular time and place. How these snippets are represented in the brain is the topic of this article.

To address this question, two types of experimental paradigms have been used: Using spatial foraging paradigms, with little emphasis on temporal structure, John O'Keefe discovered place cells (O'Keefe \& Dostrovsky, 1971), hippocampal neurons that fire at particular places in space. This led to several decades of exploration of neural coding of space, culminating with the discovery by May-Britt and Edvard Moser (Hafting, Fyhn, Molden, Moser, \& Moser, 2005) of a grid cell system of medial entorhinal cortex (EC) neurons that establish a metric of space by firing at the vortices of a spatial mesh mapped onto the spatial context. In a separate line of research, using trialbased paradigms, rich in temporal structure but minimizing spatial exploration, Howard Eichenbaum and colleagues (Eichenbaum, 2014) discovered that hippocampal time cells fired at particular time intervals during the task (reviewed in Hasselmo, Hinman, Dannenberg, \& Stern, 2017).

Catalin V. Buhusi

Catalin.Buhusi@usu.edu

1 Interdisciplinary Program in Neuroscience, Department of Psychology, USTAR BioInnovations Center, Utah State University, Logan, UT 84322, USA
To reconcile the findings obtained in space-rich (foraging) and time-rich (trial-based) paradigms, Tsao et al. (2018) recorded lateral EC (LEC) neuron activity when rats explored several spatial contexts (episodes) in sequence. The results indicated that LEC neurons exhibit a wide range of dynamic activity: Some increase (or decrease) firing throughout the session, others fire only during an episode but not in the interim, while yet others exhibit complex patterns of firing. Most importantly, none of these cells alone seems to code for the sequence of episodes, but rather all cells, as a population, code for the sequence of episodes, in a multidimensional space in which they make a trajectory linking each space-time event in the session at multiple scales: second to second and minute to minute (within episodes), and across tens of minutes (between episodes). Although in this sequential-episode paradigm the population trajectory encodes both spatial and temporal information, when rats perform in a spatial-alternation task (multiple similar trials with limited spatial attributes), the population code is biased toward coding for temporal but not for spatial information. This reconciles the conflicting studies on the roles of place cells, grid cells, and time cells, suggesting that all are informed by complex LEC population trajectories that exhibit two useful properties that make them excellent candidates for episodic memory codes: They are readily available without prior training, and they capture experience at multiple time scales (Tsao et al., 2018).

Yet a critical question remains unanswered: Since a trajectory can be pursued in both past $\rightarrow$ future and future $\rightarrow$ past orders, how does one know, upon recall, in what direction to read the trajectory? Elsewhere, I have argued that neural population coding can provide information on intervals but not on their temporal order (Buhusi, Oprisan, \& Buhusi, 2018). For example, early timing models conveniently assumed that time is coded by 
the number of pulses in an accumulator. Since numbers convey both metric and ordinal information, these models might explain both interval timing and episodic timing. In contrast, population coding is capable of conveying metric (interval) time information, but not temporal order, because the state trajectory of the system can be pursued in both past $\rightarrow$ future and future $\rightarrow$ past orders. Supplemental assumptions (e.g., pastfuture asymmetries) need to be added to population codes in order to convey episodic time (Buhusi et al., 2018).

In Tsao et al. (2018), temporal information was extracted from the population code using a complex decoder technique, which indicated that each episode predicted the adjacent episodes. This indicates that the population code makes a trajectory, but it does not answer the question of how temporal order is extracted from this code. Indeed, in Tsao et al.'s study, a past-future asymmetry was built directly into the decoder, by assuming that time flows in a particular direction. Without this assumption, upon decoding the population code, episodes were linked in a trajectory that could be read in both the past $\rightarrow$ future and the reverse order. The decoder would work in the same way and would reach the same trajectory if it were applied to the same neural recordings played in reverse.

Cues as to how the correct temporal order can be extracted from episodic experience are provided in a practical manner, by a simple experiment using the iconic "Strawberry Fields Forever" video (Martin, 1967). The video is made up of episodes, some shown in real time and some in reverse. The soundtrack is also built by foreground real-time music superimposed onto a background track played in reverse. Such mixing techniques are common in today's digital world, but at the time that the video was created, the analog techniques used to mix the real-time and reverse tracks were so innovative that this video became a landmark feature at the Museum of Modern Art in New York. Upon watching the video, it immediately becomes evident that in some episodes characters move awkwardly and that the iconic background sounds are not produced by regular instruments, such that one immediately realizes that in these episodes temporal order has been reversed (while interval time is unchanged). How does one decode the correct video trajectory?

Several answers come to mind. For one, associative learning could allow one to dissociate the likely trajectory (Paul McCartney jumping off a tree branch) from the unlikely trajectory (Paul McCartney effortlessly jumping from the ground onto a 6-foot-high tree branch). Clearly, an alien unfamiliar with how Earthlings move, with Earth gravity, and how high Earthligs could jump could not figure out that the temporal order of this episode was reversed. Alternatively, temporal asymmetries created by chemical and/or circuit-level processes in our brain may construe the reading of the population code in only one direction. For example, circuit-level asymmetries might exist with regard to encoding/decoding episodic trajectories (Roy et al., 2017), making it easy to extract temporal order. Evolution may have biased our brain cells and circuits to perceive temporal order in sync with the temporal structure of the universe, and against other possible order schemes, in the same way that evolution has built in our visual system a closefar asymmetry that is revealed by the Ponzo illusion, to help us integrate object size within the spatial structure of the world.

Either way, temporal order and episodic time are the intersection of associative learning and temporal learning, representing associative connections between temporal events without the very precise metric of interval timing. We are at the brink of understanding the neural underpinnings of how space and time weave during episodic memory formation and retrieval. Theoretical modeling of place cells, grid cells, and time cells was rewarded by the discovery of boundary cells, head direction cells, splitter cells, and so forth, suggesting a rich brain representation in which the hippocampus codes path length and the EC codes the distance to the goal, while septum informs both grid cells and time cells, but not place cells (reviewed in Hasselmo et al., 2017). Through theoretical and experimental efforts, new findings will surely follow to address questions related to how objects are linked and coded into episodic memory, how temporal order is established, and how this vast and complex pattern of information can be seamlessly coded and decoded in our brain, on the fly, in real time.

\section{References}

Buhusi, C. V., Oprisan, S. A., \& Buhusi, M. (2018). Biological and cognitive frameworks for a mental timeline. Frontiers in Neuroscience, 12, 377. https://doi.org/10.3389/fnins.2018.00377

Eichenbaum H. (2014). Time cells in the hippocampus: a new dimension for mapping memories. Nature Reviews Neuroscience, 15(11), 73244. https://doi.org/10.1038/nrn3827

Hafting, T., Fyhn, M., Molden, S., Moser, M.-B., \& Moser, E. I. (2005). Microstructure of a spatial map in the entorhinal cortex. Nature, 436, 801-806. https://doi.org/10.1038/nature03721

Hasselmo, M. E., Hinman, J. R., Dannenberg, H., \& Stern, C. E. (2017). Models of spatial and temporal dimensions of memory. Current Opinion in Behavioral Sciences 17, 27-33. https://doi.org/10. 1016/j.cobeha.2017.05.024

Martin, G. (Producer). (1967). The Beatles: Strawberry fields forever. EMI Studios, Abbey Road: Vevo LLC. Retrieved from https:// www.youtube.com/watch?v=HtUH9z_Oey8

O'Keefe, J., \& Dostrovsky, J. (1971). The hippocampus as a spatial map: Preliminary evidence from unit activity in the freely moving rat. Brain Research, 34, 171-175. https://doi.org/10.1016/00068993(71)90358-1

Roy, D. S., Kitamura, T., Okuyama, T., Ogawa, S. K., Sun, C., Obata, Y., . .. Tonegawa, S. (2017). Distinct neural circuits for the formation and retrieval of episodic memories. Cell, 170, e1019:1000-1012. https:// doi.org/10.1016/j.cell.2017.07.013

Tsao, A., Sugar, J., Lu, L., Wang, C., Knierim, J. J., Moser, M. B., \& Moser, E. I. (2018). Integrating time from experience in the lateral entorhinal cortex. Nature, 561, 57-62. https://doi.org/10.1038/ s41586-018-0459-6

Publisher's note Springer Nature remains neutral with regard to jurisdictional claims in published maps and institutional affiliations. 